\title{
REFLEXIVE REPRESENTABILITY AND STABLE METRICS
}

\author{
ITAÏ BEN YAACOV, ALEXANDER BERENSTEIN, AND STEFANO FERRI
}

\begin{abstract}
It is well-known that a topological group can be represented as a group of isometries of a reflexive Banach space if and only if its topology is induced by weakly almost periodic functions (see Sht94, Meg01b and Meg07]). We show that for a metrisable group this is equivalent to the property that its metric is uniformly equivalent to a stable metric in the sense of Krivine and Maurey (see [KM81]). This result is used to give a partial negative answer to a problem of Megrelishvili.
\end{abstract}

\section{INTRODUCTION}

In this paper we shall show that, for a topological group $G$ equipped left-invariant metric, the topology of $G$ is induced by weakly almost periodic functions if and only if its metric is uniformly equivalent to a stable one. This result allows to give simple proofs that several classical Banach spaces cannot be represented as isometries of a reflexive Banach space.

We start by introducing some definitions and terminology. Let $G$ be a topological group and let $C_{b}(G)$ denote the algebra of continuous, bounded, complex-valued functions on $G$ equipped with the supremum norm.

Definition 1.1. A function $f \in C_{b}(G)$ is said to be weakly almost periodic if the set of left translates $\left\{f_{x}: x \in G\right\}$ (where $f_{x}(y)=f(x y)$ ) is a relatively weakly compact subset of $C_{b}(G)$.

The set of all weakly almost periodic functions on $G$ forms a closed subalgebra of $C_{b}(G)$ which we shall denote by $\operatorname{WAP}(G)$. The functions in $\operatorname{WAP}(G)$ can be characterised by the following criterion due to Grothendieck (see Gro52 for a proof):

Fact 1.2 (Grothendieck's Criterion). A function $f \in C_{b}(G)$ is weakly almost periodic if and only if for all sequences $\left(s_{n}\right)_{n},\left(t_{m}\right)_{m}$ in $G$, we have

$$
\lim _{n \rightarrow \infty} \lim _{m \rightarrow \infty} f\left(s_{n} t_{m}\right)=\lim _{m \rightarrow \infty} \lim _{n \rightarrow \infty} f\left(s_{n} t_{m}\right)
$$

whenever the limits exist. Equivalently, $f \in \operatorname{WAP}(G)$ if and only if for all sequences $\left(s_{n}\right)_{n},\left(t_{m}\right)_{m}$ in $G$ and ultrafilters $\mathcal{U}, \mathcal{V}$ on $\mathbb{N}$ we have

$$
\lim _{n, \mathcal{U}} \lim _{m, \mathcal{V}} f\left(s_{n} t_{m}\right)=\lim _{m, \mathcal{V}} \lim _{n, \mathcal{U}} f\left(s_{n} t_{m}\right) .
$$

We recall that, given a countable set $\left\{x_{n}: n \in \mathbb{N}\right\}$ in a topological space and an ultrafilter $\mathcal{U}$ on $\mathbb{N}$, we write $\lim _{n, \mathcal{U}} x_{n}=L$ if for every neighbourhood $N$ of $L$ the set $\left\{n \in \mathbb{N}: x_{n} \in N\right\}$ belongs to $\mathcal{U}$.

\footnotetext{
2000 Mathematics Subject Classification. 43A60; 22A10; 46B20.

Key words and phrases. Weakly almost periodic function, weakly almost periodic compactification, reflexively representable group, stable Banach space.

The first author is supported by ANR chaire d'excellence junior THEMODMET (ANR-06-CEXC-007) and by Marie Curie research network ModNet. The second author is supported by the aforementioned ANR grant and by a research grant of the Facultad de Ciencias de la Universidad de los Andes. The third author thanks the Facultad de Ciencias de la Universidad de los Andes for the support received via the Proyecto Semilla "Large groups and semigroups and their actions".
}

Revision 927 of 10 June 2009. 
It is a classical result due to A. I. Shtern (see [Sht94]) that every compact semitopological group (and so a fortiori every compact topological group) can be represented as a group of isometries of a reflexive Banach space equipped with the weak operator topology. We recall that the weak operator topology on the space $B(E)$ of all continuous linear endomorphisms of a Banach space $E$ is the weak topology induced by the maps $\psi_{v, f}(T)=\langle f, T v\rangle$; where $v \in E, f \in E^{*}$, and $\langle\cdot, \cdot\rangle$ is the dual pairing between $E$ and $E^{*}$.

Another classical result states that the weak operator topology on the unitary group of a Hilbert space coincides with its strong operator topology (the strong topology on $B(E)$ is defined as the weak topology induced by the maps $\left.\rho_{v}(T)=T(v), v \in E\right)$. The same result is true for the isometry group of any reflexive Banach space (see $\mathrm{Meg} 01 \mathrm{~b}]$ ).

Moreover, on a compact semitopological semigroup every function on $C_{b}(G)$ is weakly almost periodic. The following theorem (see Sht94, Meg01b and Meg02]) relates the richness of the algebra WAP $(G)$ to the property that $G$ can be embedded into the isometry group of a reflexive Banach space.

Fact 1.3. Let $G$ be a topological group. Then there is a topological isomorphism of $G$ into the isometry group of a reflexive Banach space with the weak (or the strong) operator topology if and only if the topology of $G$ is induced by $\operatorname{WAP}(G)$, that is if and only if $\operatorname{WAP}(G)$ separates the identity e of $G$ from every closed subset not containing $e$.

If a group satisfies the two equivalent properties of the previous theorem it is said to be reflexively representable.

When $G$ is locally compact, the left regular representation $\lambda(g)(f(x))=f\left(g^{-1} x\right), g \in G, f \in L^{p}(G, \mu)$, $p \geq 2$, where $\mu$ is the left Haar measure, establishes a topological isomorphism of $G$ into the isometry group of the reflexive Banach space $L^{p}(G)$. Locally compact groups are therefore reflexively representable (it can also be proved directly that locally compact groups have enough weakly almost periodic functions to separate points from closed sets). On the opposite extreme we find the group $H_{+}[0,1]$ of all orientation preserving homeomorphisms of the interval $[0,1]$ with the topology of uniform convergence on compact subsets. It was proved by Megrelishvili (Meg01a) that this group has no nonconstant weakly almost periodic function.

Fact 1.4. Let $G$ be a topological group, $f \in \operatorname{WAP}(G)$. Then $f$ is both right and left uniformly continuous, meaning that for every $\varepsilon>0$ there exists a neighbourhood $e \in U$ such that $|f(x)-f(x g)|,|f(x)-f(g x)|<\varepsilon$ for every $x \in G, g \in U$.

Proof. Usp02, discussion following Theorem 5.3.

We pass now to the definition of a stable metric.

Definition 1.5. We say that a metric $d(x, y)$ is stable if for all bounded sequences $\left(s_{n}\right)_{n},\left(t_{m}\right)_{m}$ in $G$ (meaning there is an $M$ such that $d\left(e, s_{n}\right) \leq M, d\left(e, t_{m}\right) \leq M$ for all $n$ and all $m$ ), and for all ultrafilters $\mathcal{U}, \mathcal{V}$ on $\mathbb{N}$ we have

$$
\lim _{n, \mathcal{U}} \lim _{m, \mathcal{V}} d\left(s_{n}, t_{m}\right)=\lim _{m, \mathcal{V}} \lim _{n, \mathcal{U}} d\left(s_{n}, t_{m}\right) .
$$

Thus, for a bounded semigroup with an invariant metric $d$, the metric $d$ is stable if and only if the function $f(x)=d(e, x)$ is weakly almost periodic.

In what follows we shall study the relation between stable metrics and reflexive representability for metrisable groups. In particular, we shall prove that for metrisable groups, being reflexively representable is the same as having their metric uniformly equivalent to a stable one.

The strategy of the proof will be to construct a stable pre-metric (defined below) using a Urysohn technique and then to compose it with a suitable function to make it a stable metric. In the next section we shall concentrate only on how to construct this function. 


\section{Correction of triangle Deficiency}

In this section we isolate a tool which has already been used by the first author in Ben05, Ben]. This tool allows one to extract, by a continuous manipulation, a metric from a pre-metric, namely, from a binary function which would be a metric if not for the fact that it fails the triangle inequality (modulo the additional assumption of local continuity).

Recall that a function $f$ from a topological space $X$ to $\mathbb{R}$ is upper semi-continuous if for every $t$ the set $\{x \in X: f(x)<t\}$ is open in $X$. Equivalently, if whenever $f(x)<t$ there exists a neighbourhood $U$ of $x$ such that $f \uparrow_{U}<t$.

Definition 2.1. Let $g:\left(\mathbb{R}^{+}\right)^{2} \rightarrow \mathbb{R}^{+}$be a symmetric, weakly increasing (i.e., non decreasing) function, satisfying $g(0, v) \leq v$ for all $v$.

(i) We say that $g$ is a strong TD function if in addition it is upper semi-continuous.

(ii) We say that $g$ is a TD function if we only know that for every $v<t$ there is $\delta>0$ such that $g(\delta, v+\delta)<t$

Notice that a weakly increasing function $g:\left(\mathbb{R}^{+}\right)^{2} \rightarrow \mathbb{R}^{+}$is upper semi-continuous if and only if whenever $g(u, v)<t$ there is $\delta>0$ such that $g(u+\delta, v+\delta)<t$. Thus strong TD implies TD. Conversely, every TD function gives rise to a strong TD function as follows.

Lemma 2.2. Assume $g$ is a TD function and let

$$
\tilde{g}(u, v)=\inf \left\{g\left(u^{\prime}, v^{\prime}\right): u^{\prime}>u, v^{\prime}>v\right\} .
$$

Then $\tilde{g}$ is a strong TD function and $g \leq \tilde{g}$.

Proof. Clearly $\tilde{g}$ is symmetric and weakly increasing. Moreover, if $\tilde{g}(u, v)<t$ there must exist by definition $\delta>0$ such that $g(u+2 \delta, v+2 \delta)<t$, whence $\tilde{g}(u+\delta, v+\delta)<t$. Thus $g$ is upper semicontinuous. By assumption, for every $v<t$ there is $\delta>0$ such that $g(\delta, v+\delta)<t \Longrightarrow \tilde{g}(0, v)<t$. Thus $\tilde{g}(0, v) \leq v$, as desired. The inequality $g \leq \tilde{g}$ follows directly from the assumption that $g$ is weakly increasing.

Definition 2.3. A pre-metric on a space $X$ is a function $h: X^{2} \rightarrow \mathbb{R}^{+}$satisfying:

(i) Reflexivity: $h(x, y)=0 \Longleftrightarrow x=y$.

(ii) Symmetry: $h(x, y)=h(y, x)$.

We say that a pre-metric is locally continuous if for every $\varepsilon>0$ there is $\delta>0$ such that:

$$
h(x, y)<\delta \Longrightarrow|h(x, z)-h(y, z)|<\varepsilon .
$$

We say that two pre-metrics $h_{1}$ and $h_{2}$ are uniformly equivalent if for every $\varepsilon>0$ there is $\delta>0$ such that:

$$
h_{i}(x, y)<\delta \Longrightarrow h_{j}(x, y)<\varepsilon \quad i, j \in\{1,2\}
$$

A metric is always a pre-metric, and two metrics are uniformly equivalent as pre-metrics if and only if each is uniformly continuous with respect to the other. A pre-metric $h$ is a metric if and only if it satisfies the triangle inequality. To every pre-metric $h$ we may attach a function which measures its triangle deficiency (TD):

$$
\operatorname{TD}_{h}(u, v)=\sup \{h(x, z): x, y, z \in X, h(x, y) \leq u, h(y, z) \leq v\} .
$$

Indeed, $h$ satisfies the triangle inequality if and only if $\operatorname{TD}_{h}(u, v) \leq u+v$.

Lemma 2.4. Let $h$ be a locally continuous pre-metric on $X$. Then $\mathrm{TD}_{h}$ is a TD function. 
Proof. Clearly $\mathrm{TD}_{h}$ is symmetric, weakly increasing and satisfies $\operatorname{TD}_{h}(0, v) \leq v$. It remains to show that for every $v<t$ there is $\delta>0$ such that $\operatorname{TD}_{h}(\delta, v+\delta)<t$. By local continuity there is $\delta>0$ such that $h(x, y)<\delta \Longrightarrow|h(x, z)-h(y, z)|<\frac{t-v}{2}$ for all $x, y, z \in X$. We may also assume that $\delta<\frac{t-v}{2}$.

Assume now that $h(x, y) \leq \delta$ and $h(y, z) \leq v+\delta<\frac{t+v}{2}$. By choice of $\delta$ we have $h(x, z)<h(y, z)+$ $\frac{t-v}{2}<t$. Thus $\operatorname{TD}_{h}(\delta, v+\delta)<t$, as desired.

Definition 2.5. A correction function for $g:\left(\mathbb{R}^{+}\right)^{2} \rightarrow \mathbb{R}^{+}$is a continuous weakly increasing function $f: \mathbb{R}^{+} \rightarrow \mathbb{R}^{+}$satisfying:

(i) $f(t)=0 \Longleftrightarrow t=0$.

(ii) $f \circ g(u, v) \leq f(u)+f(v)$.

Lemma 2.6. Let $X$ be a space, $h$ a locally continuous pre-metric on $X$ and $f$ a correction function for $\mathrm{TD}_{h}$. Then $d_{1}=f \circ h$ is a metric on $X$, uniformly equivalent to $h$.

Proof. To see that $f \circ h$ is a metric all we need to check is the triangle inequality. Let $x, y, z \in X$ and let $u=h(x, y), v=h(y, z), w=h(x, z)$. Then $w \leq \operatorname{TD}_{h}(u, v)$, whereby $f(w) \leq f \circ \operatorname{TD}_{h}(u, v) \leq f(u)+f(v)$, as desired.

Now let $\varepsilon>0$. The correction function $f$ is continuous and thus uniformly continuous on the compact $[0,1]$, so there exists $\delta>0$ such that $t<\delta \Longrightarrow f(t)<\varepsilon$. Conversely, $f(\varepsilon)>0$ and $f(t)<f(\varepsilon) \Longrightarrow t<\varepsilon$. Thus $h$ and $f \circ h$ are uniformly equivalent.

Lemma 2.7. Let $g$ be a TD function. Then there exists a correction function $f$ for $g$.

Proof. Let $g$ be a TD function and let $\tilde{g}$ be as in Lemma 2.2. Then $\tilde{g}$ is strong TD and $g \leq \tilde{g}$. If we $\tilde{g}$ admits a correction function $f$ then $f \circ g \leq f \circ \tilde{g}$, whereby $f$ is a correction function for $g$ as well. It is therefore enough to prove that every strong TD function $g$ admits a correction function.

Let $D=\left\{\frac{k}{2^{n}}: n \in \mathbb{N}, 0<k \leq 2^{n}\right\}$ be the set of dyadic numbers in $\left.] 0,1\right]$. We shall proceed with a Urysohn style construction of $f$, choosing open sets $U_{q} \subseteq \mathbb{R}^{+}$for $q \in D$ satisfying $U_{q} \subseteq\{t: f(t)<q\} \subseteq$ $\{t: f(t) \leq q\} \subseteq U_{q^{\prime}}$ where $q<q^{\prime}$. Since we wish $f$ to be weakly increasing, each such open set will be of the form $\left[0, r_{q}\left[\right.\right.$. It is therefore enough to choose the right end-points $\left\{r_{q}\right\}_{q \in D}$. We proceed as in Ben05, Lemma 2.19], so that for all $q, q^{\prime} \in D$ we have:

(a) $0<r_{q} \leq q$,

(b) If $q<q^{\prime}$ then $r_{q}<r_{q^{\prime}}$ and $g\left(r_{q}, r_{q^{\prime}-q}\right)<r_{q^{\prime}}$.

We choose $r_{q}$ for $q=\frac{k}{2^{n}} \in D$ by induction on $n$. For $n=0$ we choose $r_{1}=1$, noticing that all the requirements hold. Assume now that $r_{q}$ has already been chosen for all $q \in D_{n}=\left\{\frac{k}{2^{n}}: 0<k \leq 2^{n}\right\}$, and we wish to choose $r_{q}$ for $q \in D_{n+1} \backslash D_{n}$, i.e., for $q=\frac{k}{2^{n+1}}, 0<k<2^{n+1}$ odd. It will be convenient to write $q^{-}=q-\frac{1}{2^{n+1}}$ and $q^{+}=q+\frac{1}{2^{n+1}}$, noticing that $q^{+} \in D_{n}$ and $q^{-} \in D_{n} \cup\{0\}$.

In case $q \geq \frac{3}{2^{n+1}}$ we have $q^{ \pm} \in D_{n}$. Let:

$$
\begin{aligned}
& s_{q, q^{\prime}}=\sup \left\{s \leq 1: g\left(s, r_{q^{\prime}}\right)<r_{q^{-}+q^{\prime}}\right\}, \\
& s_{q}=\min \left\{q, r_{q^{+}}, s_{q, q^{\prime}}: q^{\prime} \in D_{n} \cap\left[0,1-q^{-}\right]\right\}, \\
& r_{q}=\frac{r_{q^{-}}+s_{q}}{2} .
\end{aligned}
$$

For $q^{\prime} \in D_{n} \cap\left[0,1-q^{-}\right]$we have $g\left(r_{q^{-}}, r_{q^{\prime}}\right)<r_{q^{-}+q^{\prime}}$ by the induction hypothesis. Since $g$ is upper semi-continuous we obtain $r_{q^{-}}<s_{q, q^{\prime}}$, and thus $r_{q^{-}}<r_{q}<s_{q} \leq r_{q^{+}}$. 
Let us now consider the case $q=\frac{1}{2^{n+1}}$. For $q^{\prime} \in D_{n+1} \cap[2 q, 1]$ we have already chosen $r_{q^{\prime}}$ and we may define:

$$
\begin{aligned}
& s_{q, 0}=\sup \left\{s \leq 1: g\left(s, \frac{1}{2} r_{2 q}\right)<r_{2 q}\right\}, \\
& s_{q, q^{\prime}}=\sup \left\{s \leq 1: g\left(s, r_{q^{\prime}}\right)<r_{q+q^{\prime}}\right\}, \\
& s_{q}=\min \left\{q, r_{2 q}, s_{q, 0}, s_{q, q^{\prime}}: q^{\prime} \in D_{n+1} \cap[2 q, 1-q]\right\}, \\
& r_{q}=\frac{1}{2} s_{q} .
\end{aligned} \quad q^{\prime} \in D_{n+1} \cap[2 q, 1-q]
$$

By assumption on $g$ we have $g\left(0, \frac{1}{2} r_{2 q}\right) \leq \frac{1}{2} r_{2 q}<r_{2 q}$, whereby $s_{q, 0}>0$. Similarly, if $q^{\prime} \in D_{n+1} \cap[2 q, 1-q]$ then $g\left(0, r_{q^{\prime}}\right) \leq r_{q^{\prime}}<r_{q+q^{\prime}}$, so again $s_{q, q^{\prime}}>0$. Thus $0<r_{q}<s_{q} \leq r_{q^{+}}$.

Let us check that (a) and (b) hold. Indeed, $r_{q}<s_{q} \leq q$ and $r_{q^{-}}<r_{q}<r_{q^{+}}\left(\right.$where $r_{0}=0$ ), ensuring that $q \mapsto r_{q}$ is strictly positive and strictly increasing on $D_{n+1}$. We are left with checking that if $q, q^{\prime} \in D_{n+1}, q<q^{\prime}$, then $g\left(r_{q}, r_{q^{\prime}-q}\right)<r_{q^{\prime}}$. Possibly exchanging $q$ with $q^{\prime}-q$ we may assume that $q \in D_{n+1} \backslash D_{n}$. Les us consider cases:

(i) $q \geq \frac{3}{2^{n+1}}, q^{\prime}-q \in D_{n}$. Since $r_{q}<s_{q, q^{\prime}-q}$ we have by (11):

$$
g\left(r_{q}, r_{q^{\prime}-q}\right)<r_{q^{-}+\left(q^{\prime}-q\right)}<r_{q^{\prime}} .
$$

(ii) $q \geq \frac{3}{2^{n+1}}, q^{\prime}-q \in D_{n+1} \backslash D_{n}$. Since $r_{q}<s_{q,\left(q^{\prime}-q\right)^{+}}$we have by (11):

$$
g\left(r_{q}, r_{q^{\prime}-q}\right) \leq g\left(r_{q}, r_{\left(q^{\prime}-q\right)^{+}}\right)<r_{q^{-}+\left(q^{\prime}-q\right)^{+}}=r_{q^{\prime}} .
$$

(iii) $q=\frac{1}{2^{n+1}}, q^{\prime}>2 q$. Since $r_{q}<s_{q, q^{\prime}-q}$ we have by (3):

$$
g\left(r_{q}, r_{q^{\prime}-q}\right)<r_{q+\left(q^{\prime}-q\right)}=r_{q^{\prime}} .
$$

(iv) $q=\frac{1}{2^{n+1}}, q^{\prime}=2 q$. Since $r_{q}<s_{q, 0}$ and $r_{q} \leq \frac{1}{2} r_{2 q}$ we have by (2):

$$
g\left(r_{q}, r_{q}\right) \leq g\left(r_{q}, \frac{1}{2} r_{2 q}\right)<r_{2 q} .
$$

We have thus verified that all the requirement hold and the inductive construction may proceed.

We define $f: \mathbb{R}^{+} \rightarrow[0,1]$ by

$$
f(t)=\sup \left\{q \in D: r_{q}<t\right\} \quad(\text { where } \sup \varnothing=0),
$$

so in particular, $f$ is continuous on the left. Since $q \mapsto r_{q}$ is strictly increasing, this definition of $f$ agrees with

$$
f(t)=\inf \left\{q \in D: r_{q}>t\right\} \quad(\text { where } \inf \varnothing=1),
$$

whereby $f$ is also continuous on the right, and thus continuous. It is clear that $f$ is weakly increasing (it is constant on $[1, \infty)$ ) and that $f(0)=0$. On the other hand, if $f(t)=0$ then $t<r_{q} \leq q$ for all $q \in D$, so $t=0$. Finally, assume that $f(u)+f(v)<t$. If $t>1$ then $f \circ g(u, v) \leq 1<t$ directly. Otherwise, we have $f(u)<t_{1}, f(v)<t_{2}=t-t_{1}$. By definition of $f$ there are $q_{1}<t_{1}$ and $q_{2}<t_{2}$ in $D$ such that $r_{q_{1}}>u, r_{q_{2}}>v$. Then:

$$
g(u, v) \leq g\left(r_{q_{1}}, r_{q_{2}}\right)<r_{q_{1}+q_{2}} \quad \Longrightarrow \quad f \circ g(u, v) \leq q_{1}+q_{2}<t .
$$

Thus $f \circ g(u, v) \leq f(u)+f(v)$, and $f$ is a correction function for $g$ as desired.

Theorem 2.8. Let $X$ be a set, $h$ a pre-metric on $X$. Then $h$ is locally continuous if and only if it is uniformly equivalent to a metric on $X$. Moreover, this metric can be taken to be of the form $f \circ h$ where $f: \mathbb{R}^{+} \rightarrow \mathbb{R}^{+}$is continuous. 
Proof. It is easy to check that if $h$ is uniformly equivalent to a metric $d$ on $X$ then it is locally continuous. Conversely, assume $h$ is locally continuous. Then $\mathrm{TD}_{h}$ is a TD function by Lemma 2.4. By Lemma 2.7 there exists a correction function $f$ for $\mathrm{TD}_{h}$. Finally, by Lemma 2.6. $f \circ h$ is a metric uniformly equivalent to $h$.

\section{STABility AND WAP-FUnCtions}

We start the section with a lemma which will allow us to consider only bounded metrics in our proofs.

Lemma 3.1. Let $G$ be a metric group with a stable metric $d$, then $\delta(x, y):=\frac{d(x, y)}{1+d(x, y)}$ is also stable.

Proof. Consider now two sequences, call them $\left(a_{n}\right)_{n}$ and $\left(b_{m}\right)_{m}$, of elements of $G$ and let $\mathcal{U}$ and $V$ be two non-principal ultrafilters on $\mathbb{N}$. We want to show that

$$
\lim _{n, \mathcal{U}} \lim _{m, \mathcal{V}} \delta\left(a_{n}, b_{m}\right)=\lim _{m, \mathcal{V}} \lim _{n, \mathcal{U}} \delta\left(a_{n}, b_{m}\right) .
$$

Given $N \in \mathbb{N}$ define $U_{N}:=\left\{n \in \mathbb{N}: d\left(e, a_{n}\right)<N\right\}$ and $V_{N}:=\left\{m \in \mathbb{N}: d\left(e, b_{m}\right)<N\right\}$. Let also $\varepsilon>0$ and let $L=1 / \varepsilon$, so $x \geq L$ implies $x /(1+x)>1-\varepsilon$. We proceed by cases:

Case 1: There is $N \in \mathbb{N}$ such that $U_{N} \in \mathcal{U}$ and $V_{N} \in \mathcal{V}$.

Since $d(x, y)$ is stable, we get that

$$
\lim _{n, \mathcal{U}} \lim _{m, \mathcal{V}} d\left(a_{n}, b_{m}\right)=\lim _{m, \mathcal{V}} \lim _{n, \mathcal{U}} d\left(a_{n}, b_{m}\right) .
$$

On the other hand, since $f(x)=x /(1+x)$ is uniformly continuous, we get

$$
\lim _{n, \mathcal{U}} \lim _{m, \mathcal{V}} \delta\left(a_{n}, b_{m}\right)=\lim _{n, \mathcal{U}} \lim _{m, \mathcal{V}} f\left(d\left(a_{n}, b_{m}\right)\right)=\lim _{m, \mathcal{V}} \lim _{n, \mathcal{U}} f\left(d\left(a_{n}, b_{m}\right)\right)=\lim _{m, \mathcal{V}} \lim _{n, \mathcal{U}} \delta\left(a_{n}, b_{m}\right) .
$$

Case 2: There is $N \in \mathbb{N}$ such that $U_{N} \in \mathcal{U}$ but that for all $M>0$ we have $V_{M} \notin \mathcal{V}$.

For $n \in \mathbb{N}$ we have

$$
\left\{m \in \mathbb{N}: d\left(a_{n}, b_{m}\right) \geq L\right\} \supseteq\left\{m \in \mathbb{N}: d\left(b_{m}, e\right) \geq L+d\left(e, a_{n}\right)\right\} \in \mathcal{V},
$$

whereby

$$
\lim _{m, \mathcal{V}} \delta\left(a_{n}, b_{m}\right) \in[1-\varepsilon, 1]
$$

and thus

$$
\lim _{n, \mathcal{U}} \lim _{m, \mathcal{V}} \delta\left(a_{n}, b_{m}\right) \in[1-\varepsilon, 1] .
$$

On the other hand, assume $m \notin V_{N+L}$. Then

$$
\left\{n \in \mathbb{N}: d\left(a_{n}, b_{m}\right)>L\right\} \supseteq\left\{n \in \mathbb{N}: d\left(a_{n}, e\right)<N\right\} \in \mathcal{U},
$$

$\mathrm{SO}$

$$
\lim _{n, \mathcal{U}} \delta\left(a_{n}, b_{m}\right) \in[1-\varepsilon, 1]
$$

and since $\left(V_{N+L}\right)^{c} \in \mathcal{V}$ :

$$
\lim _{m, \mathcal{V}} \lim _{n, \mathcal{U}} \delta\left(a_{n}, b_{m}\right) \in[1-\varepsilon, 1] .
$$

Since $\varepsilon>0$ is arbitrary, we obtain

$$
\lim _{n, \mathcal{U}} \lim _{m, \mathcal{V}} \delta\left(a_{n}, b_{m}\right)=1=\lim _{m, \mathcal{V}} \lim _{n, \mathcal{U}} \delta\left(a_{n}, b_{m}\right) .
$$

Case 3: For all $N \in \mathbb{N}$ we have $U_{N} \notin \mathcal{U}$ and $V_{N} \notin \mathcal{V}$. 
Just like in the previous case, we show that

$$
\lim _{n, \mathcal{U}} \lim _{m, \mathcal{V}} \delta\left(a_{n}, b_{m}\right)=1=\lim _{m, \mathcal{V}} \lim _{n, \mathcal{U}} \delta\left(a_{n}, b_{m}\right) .
$$

We are now ready to prove the main result of our paper, namely, that a metrisable group is reflexive representable if and only if its metric is uniformly equivalent to a stable metric. We shall prove separately the two implications.

Chaatit Cha96 proved that the additive group of any stable separable Banach space can be represented as a group of isometries of a separable reflexive Banach space. First we show, applying results by Shtern (Fact 1.3), that any stable group is reflexively representable.

Proposition 3.2. Assume that $G$ is a group equipped with a left-invariant stable metric d. Then $G$ is reflexively representable.

Proof. By Lemma 3.1 we can assume without loss of generality that $d$ is bounded. By Grothendieck's Criterion (Fact 1.2), and since $d(x, y)=d\left(e, x^{-1} y\right)$ is a stable distance, the function $f(x)=d(e, x)$ is weakly almost periodic. Clearly it separates the identity from every closed set not containing it. By Fact 1.3, $G$ is reflexively representable.

Conversely,

Theorem 3.3. Let $G$ be a group equipped with a left-invariant metric, and assume that $G$ is reflexively representable. Then there is a left-invariant stable metric $\delta$ on $G$ that is equivalent to $d$.

Proof. As in the previous result, by Lemma 3.1 we may assume that $d$ is bounded, and using the results proved in the previous section it suffices to produce a left-invariant stable locally continuous pre-metric uniformly equivalent to $d$.

We now construct by induction a sequence of symmetric functions $f_{n}: G \longrightarrow[0,1]$ in $\operatorname{WAP}(G)$ (here, $f$ symmetric means that $f(g)=f\left(g^{-1}\right)$ for all $\left.g \in G\right)$ and radii $r_{n}>0$ such that the following conditions hold:

(a) $f_{n} \uparrow_{B\left(e, r_{n+1}\right)}=0$, where $B(e, r)$ denotes the open ball of radius $r$ around $e$.

(b) $f_{n} \uparrow_{G \backslash B\left(e, r_{n}\right)}=1$,

(c) $r_{n} \leq 2^{-n}$.

We start with $r_{0}=1$. Given $r_{n}$ we choose $f_{n}$ and $r_{n+1}$ as follows. First, by Fact 1.3 and our hypothesis, the algebra $\operatorname{WAP}(G)$ separates points from closed sets. Therefore there exists a function $f_{n}: G \longrightarrow[0,1]$ in $\operatorname{WAP}(G)$, such that $f_{n}\left[G \backslash B\left(e, r_{n}\right)\right]=1$ and $f_{n}(e)=0$. Possibly replacing $f_{n}$ with $\min \left(f_{n}(x), f_{n}\left(x^{-1}\right)\right)$ we may assume that $f_{n}$ is symmetric. Possibly replacing $f_{n}$ with $\left(2 f_{n}-1\right)^{+}$ we may further assume that $f_{n}$ is zero on a neighbourhood of $e$, and we may choose $r_{n+1}$ such that $f_{n} \uparrow_{B\left(e, r_{n+1}\right)}=0$. We may further require that $r_{n+1} \leq 2^{-n-1}$.

Once we are done we define

$$
h(x, y):=\sum_{n=0}^{\infty} \frac{f_{n}\left(x^{-1} y\right)}{2^{n+1}} .
$$

We claim that $h$ is a left-invariant stable locally continuous pre-metric uniformly equivalent to $d$ : - Left invariance, reflexivity $h(x, x)=0$ and symmetry $h(x, y)=h(y, x)$ follow immediately from the construction.

- Stability follows from the fact that $h$ is a uniform limit of functions in $\operatorname{WAP}(G)$ which is a norm closed sub-algebra of $C_{b}(G)$.

- By Fact 1.4 $h$ is uniformly continuous (with respect to $d$ ). Conversely assume that $h(x, y)>2^{-n}$. This means that $f_{m}\left(x^{-1} y\right)>0$ for some $m<n$, so $x^{-1} y \notin B\left(e, r_{n}\right)$, i.e., $d(x . y) \geq r_{n}$. Thus $d$ and $h$ are uniformly equivalent, and it follows that $h$ is locally continuous. 


\section{Some Applications}

In this last section we shall use the characterisation of reflexive representability we just proved to establish that the additive group of several classical Banach spaces is not reflexively representable.

Megrelishvili in [Meg07], [Meg02 and [Meg01a and Glasner and Megrelishvili in [GM08, Problem 6.11] explicitely asked whether it was possible to find an abelian group which is not reflexively representable. The problem was solved directly in [FG] using a modification of Raynaud's proof Ray83 of non uniform embeddability of $c_{0}$ into $\ell_{2}$. The same result can also be deduced from two facts: the space $c_{0}$ cannot be uniformly embedded into a reflexive Banach space (see [Kal07]) and a reflexively representable metric group always embeds uniformly into a reflexive Banach space (see Meg01b, Proposition 3.4]). A similar result also holds for the quasi-reflexive James' space $J$ : it is not uniformly embeddable into a reflexive space (see Kal07) and thus it is not reflexively representable.

In the light of our results it can now be proved very easily that $c_{0}$ is not reflexively representable. Notice that this result also answers Question 6.12 of Meg07 since we show that there exist quotients of reflexively representable groups which are not reflexively representable. This because $c_{0}$ is, as every separable Banach space, a quotient of $\ell_{1}$ which is reflexively representable (in fact, it is even unitarily representable).

Theorem 4.1. The additive group of the Banach space $c_{0}$ is not reflexively representable.

Proof. Suppose that $c_{0}$ is reflexively representable. Then its metric would be uniformly equivalent to a stable one, which is impossible by [Ray83, Théorème 5.1].

We use now our result to prove that Tsirelson's space $T$ is not reflexively representable. Since $T$ is reflexive, this gives a partial negative answer to [Meg07, Question 6.9] (see also [Meg02]), as it shows that there are metrisable groups which are uniformly embedded into a reflexive Banach space (in fact, additive groups of reflexive Banach spaces) which are not reflexively representable.

Theorem 4.2. Tsirelson's space $T$ is not reflexively representable.

Proof. By [Ray83, Théorème 4.1], if a Banach space admits a translation invariant stable metric which is uniformly equivalent to its norm-induced metric, then it contains a copy of $\ell_{p}$ for some $p$. Hence Tsirelson's space $T$ does not admit such a metric and, since the metric produced in Theorem 3.3 is clearly translation invariant, Tsirelson's space is not reflexively representable.

In a similar way it follows that Tsirelson-like spaces such as Schlumprecht's space $\mathcal{S}_{f}$ (see [BL00, Corollary 13.31]) are also not reflexively representable.

\section{REFERENCES}

[Ben] Itaï Ben Yaacov, Definability of groups in $\aleph_{0}$-stable metric structures, submitted, arXiv:0802.4286.

[Ben05] _ Uncountable dense categoricity in cats, Journal of Symbolic Logic 70 (2005), no. 3, 829-860.

[BL00] Yoav Benyamini and Joram Lindenstrauss, Geometric nonlinear functional analysis. Vol. 1, American Mathematical Society Colloquium Publications, vol. 48, American Mathematical Society, Providence, RI, 2000.

[Cha96] Fouad Chaatit, A representation of stable Banach spaces, Archiv der Mathematik 67 (1996), no. 1, 59-69.

[FG] Stefano Ferri and Jorge Galindo, Embedding a topological group into its WAP-compactification, Studia Mathematica, to appear.

[GM08] Eli Glasner and Michael G. Megrelishvili, Some new algebras of functions on topological groups arising from G-spaces Fundamenta Mathematicae 201 (2008), 1-51, arXiv:math/0608575

[Gro52] Alexandre Grothendieck, Critères de compacité dans les espaces fonctionnels généraux, American Journal of Mathematics 74 (1952), 168-186.

[Kal07] N. J. Kalton, Coarse and uniform embeddings into reflexive spaces, The Quarterly Journal of Mathematics $\mathbf{5 8}$ (2007), no. 3, 393-414.

[KM81] Jean-Louis Krivine and Bernard Maurey, Espaces de Banach stables, Israel Journal of Mathematics 39 (1981), no. 4, 273-295. 
[Meg01a] Michael G. Megrelishvili, Every semitopological semigroup compactification of the group $H_{+}[0,1]$ is trivial, Semigroup Forum 63 (2001), no. 3, 357-370.

[Meg01b] _ Operator topologies and reflexive representability, Nuclear groups and Lie groups (Madrid, 1999), Res. Exp. Math., vol. 24, Heldermann, Lemgo, 2001, pp. 197-208.

[Meg02] _ Reflexively but not unitarily representable topological groups, Proceedings of the 15th Summer Conference on General Topology and its Applications/1st Turkish International Conference on Topology and its Applications (Oxford, OH/Istanbul, 2000), Topology Proceedings, vol. 25, 2002, pp. 615-625.

[Meg07] _ Topological transformation groups: selected topics, Open Problems In Topology II (Elliott Pearl, ed.), Elsevier, 2007.

[Ray83] Yves Raynaud, Espaces de Banach superstables, distances stables et homéomorphismes uniformes, Israel Journal of Mathematics 44 (1983), no. 1, 33-52.

[Sht94] Alexander I. Shtern, Compact semitopological semigroups and reflexive representability of topological groups, Russian Journal of Mathematical Physics 2 (1994), no. 1, 131-132.

[Usp02] Vladimir Uspenskij, Compactifications of topological groups, Proceedings of the Ninth Prague Topological Symposium (2001), Topology Atlas, North Bay, ON, 2002, pp. 331-346 (electronic).

Itä̈ Ben Yaacov, Université Claude Bernard - Lyon 1, Institut Camille Jordan, 43 boulevard du 11 novembre 1918, 69622 Villeurbanne Cedex, France

$U R L:$ http://math.univ-lyon1.fr/ begnac/

Alexander Berenstein, Universidad de los Andes, Departamento de Matemáticas, Carrera 1 \# 18A-10, Bogotá, Colombia ; And

Université Claude Bernard - Lyon 1, Institut Camille Jordan, 43 Boulevard du 11 novembre 1918,69622 Villeurbanne Cedex, France

E-mail address: aberenst@uniandes.edu.co

$U R L:$ http://matematicas.uniandes.edu.co/ aberenst

Stefano Ferri Universidad de los Andes, Departamento de Matemáticas, Carrera 1 \# 18A-10, Bogotá, Colombia

E-mail address: stferri@uniandes.edu.co

$U R L:$ http://matematicas.uniandes.edu.co/ stferri/ferri.html 